\title{
Implicações das restrições alimentares na vida diária de mulheres com diabete melito gestacional
}

\author{
Implications of diet restrictions in the daily lives of women with gestational Diabetes Mellitus \\ Implicaciones de las restricciones alimentares en la vida diaria de mujeres con Diabetes \\ Mellitus en la gestación
}

\author{
Joice Moreira Schmalfuss ${ }^{I}$; Ana Lucia de Lourenzi Bonilha ${ }^{I I}$
}

\begin{abstract}
RESUMO: A dieta é um dos principais focos do tratamento das gestantes diabéticas, constituindo-se em estratégia recomendada para um acompanhamento gestacional adequado e o nascimento de um bebê saudável. Trata-se de uma pesquisa qualitativa, do tipo descritivo, que objetivou conhecer as implicações das restrições alimentares na vida diária de mulheres com diabete melito gestacional. O estudo foi realizado em um hospital universitário do município de Porto Alegre/RS, por meio de entrevistas com 25 gestantes diabéticas em acompanhamento ambulatorial, entre os meses de julho a novembro de 2010. A análise de dados foi do tipo temática. As mulheres apresentam dificuldades em seguir o plano alimentar prescrito, fato que provoca implicações negativas nas suas vidas diárias. A orientação nutricional deve ser flexível e respeitar a condição social de cada gestante. Há necessidade de elaboração de estratégias e propostas de cuidados que auxiliem essas mulheres no enfrentamento dos obstáculos relacionados à dieta alimentar.

Palavras-Chave: Diabetes gestacional; gravidez de alto risco; cuidado pré-natal; dieta para diabéticos.
\end{abstract}

\begin{abstract}
Diet is one of the main focuses of treatment for diabetic pregnant women, and constitutes a recommended strategy for appropriate gestational monitoring and healthy childbirth. This qualitative, descriptive study examined the implications of diet restrictions in the daily lives of women with gestational diabetes mellitus. The study was performed between July and November 2010 at a university hospital in the city of Porto Alegre, Rio Grande do Sul, by means of interviews of 25 diabetic pregnant women in outpatient care. Data assessment was thematic. The women had difficulty sticking to the prescribed diet plan, which has adverse implications for their daily lives. Nutritional counseling should be flexible and contemplate the social condition of each pregnant woman. Care strategies and proposals should be developed to help these women surmount diet-related obstacles. Keywords: Gestational diabetes; high-risk pregnancy; antenatal care; diet for diabetics.
\end{abstract}

RESUMEN: La dieta es un aspecto importante del tratamiento de las mujeres diabéticas embarazadas, que constituye una estrategia recomendada para un adecuado seguimiento del embarazo y el nacimiento de un hijo sano. Se trata de una pesquisa cualitativa, del tipo descriptivo que tuvo el objetivo de conocer las implicaciones de las restricciones alimentares en la vida diaria de las mujeres con Diabetes Mellitus en la gestación. El estudio fue realizado en un hospital universitario de la municipalidad de Porto Alegre/RS - Brasil, por medio de entrevistas con 25 gestantes diabéticas en acompañamiento ambulatorio, entre los meses de julio a noviembre de 2010. El análisis de datos fue del tipo temático. Las mujeres tienen dificultades para seguir el plan de la dieta prescrita, lo que provoca consecuencias negativas en sus vidas diarias. Asesoramiento nutricional debe ser flexible y respetar la condición social de cada mujer embarazada. Existe la necesidad de desarrollar estratégias y propuestas que ayuden esas mujeres a enfrentaren los obstáculos relacionados con la alimentación.

Palabras Clave: Diabetes gestacional; embarazo de alto riesgo; cuidado prenatal; dieta para diabéticos.

\section{INTRODUÇÃO}

O diabete melito gestacional (DMG) é uma das classificações ${ }^{1}$ do diabete melito (DM), no qual conceitua-se como um grupo de doenças metabólicas de etiologia múltipla caracterizado por hiperglicemia decorrente de efeitos na secreção e/ou ação da insulina $^{1,2}$ que, com maior frequência, coincide com a gravidez $z^{3}$.
Aproximadamente $7 \%$ de todas as gestações no mundo são complicadas pelo DMG, resultando em mais de 200.000 casos por ano e representando $90 \%$ dos casos dessa patologia. A prevalência pode variar de 1 a $14 \%$, dependendo da população estudada e dos testes diagnósticos empregados ${ }^{4}$. Na Espanha, a prevalência de DMG em mulheres acompanhadas em

${ }^{I}$ Mestre em Enfermagem. Especialista em Enfermagem Obstétrica. Professora Assistente do Curso de Graduação em Enfermagem da Universidade Federal da Fronteira Sul. Chapecó, Santa Catarina, Brasil.E-mail: joicemschmalfuss@gmail.com

IDDutora em Enfermagem. Professora Titular da Escola de Enfermagem e do Programa de Pós-Graduação em Enfermagem da Universidade Federal do Rio Grande do Sul. Porto Alegre, Rio Grande do Sul, Brasil. E-mail: bonilha.ana@gmail.com 
serviço de endocrinologia e nutrição e nas consultas obstétricas de alto risco foi de $4,2 \%{ }^{3}$.

No Brasil, em 2010, a prevalência de DMG em mulheres com mais de 20 anos de idade atendidas no Sistema Único de Saúde foi de 7,6\%5 . Um estudo para determinar a prevalência de DMG em grávidas atendidas em Unidades Básicas de Saúde da cidade de Vitória/ES concluiu que essa taxa foi de 5,8\% em uma amostra de 396 gestantes ${ }^{6}$. Em outro estudo realizado em Pelotas/RS, que investigou os fatores associados à ocorrência de DMG em 4.243 puérperas, a prevalência dessa patologia foi de 2,95\%?

As taxas anteriormente citadas demonstram a relevância do DMG, tanto como uma intercorrência adquirida no decorrer da gravidez, quanto como um problema de saúde pública. Essa constatação se agrava quando a doença não é controlada, podendo resultar em consequências graves para o binômio mãe-bebê.

Em função disso e visando evitar prejuízos que podem acometer o binômio, é fundamental que a mulher com DMG adote cuidados como: controle glicêmico, atividade física, acompanhamento ambulatorial com profissionais habilitados para atender gestantes de alto risco, juntamente à realização de dieta alimentar. Este último item, em especial, possui grande importância para o sucesso do tratamento do DMG, além de se constituir em estratégia recomendada para um acompanhamento gestacional adequado e para o nascimento de um bebê saudável.

Diante do exposto, surge o seguinte questionamento: quais são as implicações causadas pelas restrições alimentares na vida diária de mulheres com DMG? Desse modo, o objetivo da presente pesquisa foi conhecer as implicações das restrições alimentares na vida diária de mulheres com DMG.

\section{ReVISÃo DE LITERATURA}

Durante a gravidez, mudanças metabólicas ocorrem no organismo da mulher a fim de dar suporte ao bebê para que este cresça e se desenvolva de forma saudável ${ }^{8}$. Estas mudanças incluem a hipoglicemia de jejum, o catabolismo exagerado dos lipídios com consequente formação de corpos cetônicos e o aumento da resistência à insulina² .

A resistência à insulina torna-se aumentada à medida que a placenta cresce e aumenta a produção dos hormônios progesterona, cortisol, lactogênio placentário humano, prolactina, hormônio do crescimento e somatotrofina ${ }^{2,8}$.

A elevação da produção hormonal placentária acontece em torno da $20^{\mathrm{a}}$ semana de gestação e progride até o final da mesma. É a maior responsável pela resistência do organismo materno à produção de insulina. Em decorrência disso, mais insulina é secretada para superar essa resistência, o que acaba por causar uma descompensação glicêmica que pode afetar a mãe e o bebêt $\hat{e}^{2,8}$. Por esse motivo, o DMG costuma ser diagnosticado no final do segundo ou início do terceiro trimestre da gravidez, quando, por ação hormonal, a resistência à insulina torna-se aumentada.

O diabete na gestação, frequentemente, está associado a complicações como hipoglicemia, hiperglicemia, cetoacidose, retinopatia, nefropatia, doença hipertensiva da gestação, polidrâmnio, trabalho de parto pré-termo, parto cesáreo por distocia de ombros, anomalias congênitas (cardíacas, renais, neurológicas e gastrointestinais), diminuição do crescimento cerebral, macrossomia fetal, fratura de clavícula, lesão do plexo braquial, hipoglicemia e hiperbilirrubinemia neonatal, doença da membrana hialina e corticoterapia antenatal ${ }^{2,9-11}$.

Considerando estas complicações, o cuidado à gestante com DMG deve começar desde a primeira consulta de pré-natal, sendo obrigatório o rastreamento para a detecção da doença. Avaliações frequentes e rigorosas do profissional pré-natalista objetivam a identificação de qualquer alteração e devem se estender durante toda a gravidez, só finalizando após seis semanas do parto.

No que tange ao manejo do DMG, destaca-se que $60 \%$ das gestantes podem se manter euglicêmicas somente com a realização de dieta e atividade física, sem acarretar maiores riscos para a gravide ${ }^{5}$ e, a hiperglicemia materna deveria ser cuidadosamente controlada apenas com a dieta ${ }^{12}$.

Nessa perspectiva, um estudo que utilizou a intervenção educativa como implementação de autocuidado e aderência terapêutica concluiu que após o desenvolvimento da intervenção com um grupo de diabéticos, este obteve resultados melhores quanto à adesão ao tratamento, conhecimento da doença, estratégias para conservar o seu bem-estar e responsabilidade com a sua saúde, gerando uma nova noção nutricional quando comparado a um grupo controle (não recebeu intervenção) ${ }^{13}$.

Esse fato mostra a importância do profissional de saúde orientar a gestante acerca da sua dieta nutricional, valorizando suas queixas e dificuldades, bem como facilitando suas adaptações alimentares aos alimentos prescritos, de forma que esta obtenha êxito no seu tratamento e evite a necessidade de complementação com insulina.

Nesse contexto, um estudo que objetivou identificar os cuidados de enfermagem prestados às mulheres com DMG durante a atenção pré-natal, constatou que todos os artigos incluídos na amostra apresentaram conformidade em relação ao papel decisivo desempenhado pelo enfermeiro no atendimento prestado à gestante diabética, salientando a importância que este profissional possui em relação à prática da educação em saúde, no seu cotidiano de trabalho, de forma a realçar o autocuidado da mulher com $\mathrm{DMG}^{14}$. 
Corroborando com o exposto, outro estudo que objetivou analisar as estratégias educativas utilizadas junto às pessoas com hipertensão e DM, constatou que os profissionais mais envolvidos com o enfoque educativo são os enfermeiros e que as estratégias emancipatórias foram as mais frequentes entre os estudos publicados ${ }^{15}$.

\section{Metodologia}

Trata-se de uma pesquisa qualitativa do tipo descritivo, realizada em um hospital universitário do município de Porto Alegre/RS, considerado referência para o atendimento de gestantes de alto risco, além de realizar grupos de gestantes diabéticas semanalmente.

As participantes do estudo foram 25 mulheres com DMG em acompanhamento ambulatorial. Os critérios de inclusão foram: gestantes com 18 anos de idade ou mais; com o diagnóstico de DMG e idade gestacional acima de 20 semanas. Foram critérios de exclusão: gestantes com o diagnóstico de diabete pré-gestacional e que apresentaram a gestação interrompida ou perda fetal.

Optou-se por apenas incluir na pesquisa as grávidas com DMG, pois ao contrário do que acontece com as mulheres que possuem diabete prévio à gestação (DM tipo 1 ou 2), aquelas que se descobrem diabéticas no decorrer da gravidez, têm o acréscimo de uma condição de risco que extrapola as peculiaridades inerentes a qualquer gestação de baixo risco. A idade gestacional acima de 20 semanas também foi considerada critério de inclusão porque o DMG costuma ser diagnosticado a partir da $20^{\mathrm{a}}$ semana, quando a resistência à insulina torna-se aumentada em decorrência de hormônios produzidos pela placenta. A fim de preservar a identidade das participantes incluídas no estudo, as mesmas foram identificadas com a letra $\mathrm{G}$ para grávidas, seguidas pela numeração de 1 a 25 .

A coleta de dados foi realizada entre os meses de julho e novembro de 2010, nas segundas-feiras (dia em que ocorre o grupo), quando foram identificadas e selecionadas as gestantes que preenchessem os critérios de inclusão. A abordagem e convite foram feitos pela pesquisadora, no próprio local de atendimento das gestantes, a partir de contato verbal com as possíveis participantes. Com o aceite da mulher e assinatura do Termo de Consentimento, foi realizada uma busca em seu prontuário eletrônico e na sua carteira de pré-natal para a coleta de dados necessários e, após, procedeu-se à entrevista semiestruturada. As entrevistas tiveram uma duração média de 30 minutos, foram realizadas nas dependências da unidade de alojamento conjunto (UIO), em ambiente que garantiu a privacidade da mulher entrevistada, e registradas em um gravador digital do tipo MP3 Player.

Os dados da presente pesquisa foram analisados conforme referencial de análise de conteúdo do tipo temática proposta por Bardin ${ }^{16}$. Os dispositivos legais da Resolução do Conselho Nacional de Saúde de número 466/2012 foram respeitados nesta pesquisa ${ }^{17}$, sendo que a mesma foi conduzida de acordo com os padrões éticos exigidos e seu projeto aprovado pela Comissão Científica e Comissão de Pesquisa e Ética em Saúde do Hospital de Clínicas de Porto Alegre (HCPA) sob o número 100230/2010.

\section{Resultados e Discussão}

\section{Caracterização das gestantes}

As 25 gestantes entrevistadas tinham, em média, 29,8 anos, com idades variando entre 21 e 39 anos. Os achados da presente pesquisa estão próximos dos dados de um estudo realizado em um hospital universitário do Rio de Janeiro/RJ, que revelou a média de idade de 29,6 anos das 21 grávidas com DMG que estavam em acompanhamento ambulatorial ${ }^{18}$.

Em relação à profissão das gestantes, identificouse que 12 delas são donas de casa, 12 trabalham com prestação de serviço em atividades como diarista, cozinheira, manicure, cabelereira, secretária, entre outras, e uma é autônoma. Dados similares foram encontrados em um estudo realizado em Porto Alegre/RS, no mesmo hospital objeto da presente pesquisa, em 2001, que concluiu que, das 105 gestantes incluídas na amostra, $51,4 \%$ se dedicavam a atividades do lar e apenas $1,9 \%$ desenvolviam atividades de forma autônoma ${ }^{19}$.

Quanto à escolaridade das participantes, 10 possuíam ensino fundamental incompleto; duas, o ensino fundamental completo; três não concluíram o ensino médio, e seis o concluíram e quatro cursaram cursos técnicos, sendo que nenhuma das participantes havia cursado ensino superior. Estes dados indicam que aproximadamente um terço das gestantes entrevistadas não completou o ensino fundamental.

Em relação à situação conjugal, 21 entrevistadas convivem com o companheiro, enquanto quatro não possuem companheiro, caracterizando-se como solteiras ou separadas. Houve semelhança com os dados encontrados em outro estudo realizado no mesmo local da pesquisa, cuja maioria $(70,5 \%$, ou 74 gestantes) era casada e/ou tinha companheiro ${ }^{19}$.

\section{Implicações das restrições alimentares na vida diária de mulheres com DMG}

A dieta é um dos principais focos do tratamento da grávida diabética, sendo que orientação nutricional deve ser fornecida por uma nutricionista a fim desta indicar os valores calóricos adequados para o consumo alimentar da grávida. Em geral, a necessidade calórica estimada situa-se entre 1.800 e 2.220 calorias diárias totais $^{20}$, sendo que os valores calóricos ingeridos pelas participantes do estudo variaram entre 1.600 para o consumo mínimo e 2.300 para o consumo máximo, com uma média de 2.000 calorias diárias totais. 
Quando questionadas sobre o tipo de dieta segui$\mathrm{da}$, destacaram-se as que seguem a dieta prescrita pela nutricionista que realiza o acompanhamento ambulatorial do pré-natal de alto risco, no hospital do presente estudo. Esta afirmação foi observada nos relatos:

[...] eu sigo aquela dieta que ela (nutricionista) deu pra mim. Eu como o que está marcado lá naquela... A quantidade. (G5)

Cortei completamente o açúcar e diminuí as porções de comida, porque comia bem mais do que isso. (G16)

Só faço a dieta, não como coisa fora de hora. (G21)

Eu cortei o açúcar, eu cortei os carboidratos. (G6)

Porém, mesmo que algumas participantes tenha afirmado realizar a dieta conforme orientado no ambulatório de pré-natal de alto risco, muitas afirmaram ter dificuldades em respeitar rigorosamente as orientações nutricionais:

[...] eu não faço corretamente seis refeições por dia não. (G4)

[...] pra mim, mais dificuldade é as seis refeições por dia, porque eu não tenho o costume de comer seis refeições por dia. (G8)

Considerando que o plano alimentar deve ser dividido em frações com menor volume, horário previamente estipulado, intervalos controlados e cinco a seis refeições diárias ${ }^{21}$, a enfermeira exerce um importante papel quando verifica-se o não cumprimento ao plano alimentar prescrito. Esta deve planejar, negociar e acomodar as preferências alimentares da mulher de forma a facilitar a adaptação e seguimento à dieta que foi proposta pelo profissional que acompanha a parte nutricional da sua gestação de alto risco ${ }^{22-24}$.

Outras dificuldades com a restrição alimentar também foram relacionadas ao ambiente de trabalho das gestantes:

[...] o problema é quando eu estou no serviço, que bem dizer, eu fico o dia inteiro. Aí, lá não tem pão integral, não tem bolachinha integral, não tem leite, não tem quase nada assim. (G2)

[...] é obrigada a sair um pouco da dieta por causa do horário do trabalho. Está atendendo alguém, não pode parar naquele horário ou está fazendo alguma outra coisa. (G3)

É difícil no meu serviço. Em casa até controlo melhor, mas no serviço não tem. (G6)

As dificuldades manifestadas pelas gestantes em relação à dieta estiveram presentes das mais diversas formas, sendo que foram observados sentimentos negativos perante a impossibilidade de comer algo que gostariam, conforme constatado nas seguintes declarações:

[...] eu não posso comer o que eu quero comer. É uma tortura tu não comer o que tu quer comer. Sempre fui acostumada a comer e agora não posso comer. É muito ruim. (G10)

Eu estou achando muito ruim porque antes eu não me travava de comer nada e agora tem que ir, lá, olhar na porta da geladeira, se em tal hora se pode comer tal coisa. Não pode comer doce... (G18)

Antes eu era acostumada a comer de tudo, não tinha limites para nada [...]. E agora não... Tem dias que me dá um mal-estar, uma vontade de comer alguma coisa. E aí não pode. A parte mais difícil é essa. (G24)

No que concerne à reeducação alimentar, salienta-se que, para muitos indivíduos, o ato de comer representa um dos maiores gozos da vida, uma forma de equilibrar algo ou até mesmo uma maneira de desprezar um problema ou decepção $0^{10,12,14,15,20-24}$.

Unido ao exposto, a privação de comer alguns alimentos, às vezes, pode causar sentimentos de descontentamento nas grávidas ${ }^{24}$. Este fato, além de gerar frustração, pode resultar em dificuldades em lidar com o DMG e revolta com a doença, sendo indicado que o profissional que acompanha estas mulheres solicite um acompanhamento psicológico, a fim de ajudar a mesma a compreender a necessidade de um planejamento alimentar para um bom prognóstico materno-fetal20-24.

A possibilidade de as gestantes fracassarem na adaptação ao plano alimentar também é outro temor comum manifestado por elas ${ }^{22}$, sendo que o desejo de ingerir um alimento proibido foi relatado pelas participantes como uma vontade muito difícil de ser controlada, até mesmo por não ser comum antes da gravidez.

[...] é mais complicado, porque agora a gente sente vontade de comer coisas que a gente olhava antes e nem dava bola. (G6)

[...] eu sei que eu estou com a diabete. Daí eu sei que eu não posso comer, daí que me dá vontade de comer. É uma coisa que parece que te chama [risos]. (G9)

Ressalta-se que além do forte desejo de comer alimentos não aconselhados no tratamento nutricional, a possibilidade de fracasso também pode ocorrer por dificuldade financeira e/ou ansiedade ${ }^{23}$, fatores externos que devem ser verificados pelos profissionais que acompanham o pré-natal das gestantes diabéticas.

Bastante relacionado ao fator ansiedade, algumas participantes alegaram não conseguir deixar de comer algum alimento desejado, desrespeitando a orientação nutricional prescrita:

[...] eu como um chocolatezinho de vez em quando. Quando eu estou com muita vontade eu como. (G1)

Eu não vou negar que eu comi. Alguma coisinha sempre acaba escapando. (G6)

Às vezes dá aquela loucura de comer um doce. Eu pego, eu me sento com um pote de mumu, pego a colher e como. (G9) 
Convergindo para os achados da presente pesquisa, estudo realizado com 12 gestantes diabéticas, acompanhadas por uma Instituição de Saúde de Fortaleza/Ceará, verificou que as gestantes apresentaram déficit de autocuidado relacionado à dieta ${ }^{23}$.

Entretanto, mesmo que algumas participantes tenham manifestado dificuldades em seguir a dieta prescrita, outras referiram não comer nada além da mesma, conforme destacam as declarações:

\section{[...] fico com vontade mas não como. (G13)}

Às vezes eu posso comer uma coisinha a mais, eu não consigo comer. Até quero comer uma bolachinha, mas não consigo, eu travo. Então, a maneira que eu estou me cuidando é seguindo direitinho a dieta. (G17)

Visando atingir êxito na terapia nutricional da gestante, o ideal é que a mesma seja trabalhada inter e multidisciplinariamente de forma a permitir um ganho apropriado de peso, um controle glicêmico e a ausência de corpos cetônicos, fatores que resultarão em benefícios para a saúde materno-fetal ${ }^{21-23}$.

Ao tratar da interação social, atenção especial deve ser dispensada à gestante diabética, pois um dos fatores que pode fazer com que ela se afaste das atividades sociais é o impedimento do consumo de certos. Dessa forma, o prejuízo nos momentos de lazer foi outra importante implicação relatada pelas participantes, sendo fortemente associada à restrição alimentar:

[...] esses dias eu fui uma festa. [...] fui embora antes de cortarem o bolo. (G4)

[...] as festas, a gente tem que ir e tem que olhar, não pode comer. (G11)

Eu evito sair em certos lugares, porque eu sei que se eu for lá eu vou ficar com vontade de comer. [...] daí eu já evito, sabe, fico em casa [...] (G13)

[...] tu vai sair, jantar fora ou na casa de outra pessoa... A tua alimentação é complicada, é mais difícil. (G15)

Festa, por exemplo, vai para os aniversários, só olha. Eu não como [risos]. (G16)

Existem diversos fatores que podem influenciar o seguimento da dieta prescrita. Ressalta-se que a fim de facilitar a adesão da grávida à dieta alimentar, é recomendado que os profissionais de saúde priorizem a qualidade das informações passadas a cada mulher e procurem facilitar sua aderência à dieta, por meio de negociação e acomodação de suas preferências alimentares.

Além disso, é necessário que a dieta indicada pela nutricionista seja flexível e respeite a condição social de cada gestante ${ }^{20}$. Esses cuidados, aliados a outros, destinam-se a favorecer seu seguimento à orientação nutricional, seja pelo entendimento das informações, pelo consumo adequado de alimentos ou pela alimentos mais em conta, conforme sua condição financeira.

\section{Conclusão}

O estudo possibilitou conhecer as implicações das restrições alimentares na vida diária de mulheres com DMG. Em relação ao seguimento do plano alimentar prescrito, durante o acompanhamento no pré-natal de alto risco, participantes relataram cumprir a recomendação indicada pela nutricionista. Porém, embora haja participantes que afirmaram realizar a dieta, outras manifestaram ter dificuldades em seguir rigorosamente as orientações nutricionais, apresentando sentimentos negativos e de descontentamento perante a privação de alguns alimentos de suas preferências. Outras gestantes, inclusive, afirmaram consumir alimentos que não estão incluídos na dieta, indicando a falta de adaptação ao plano nutricional.

Em função da restrição dietética, há participantes que também relataram prejuízos relacionados aos momentos de lazer e hábitos de frequentar locais públicos como shoppings, festas, casas de amigos, entre outros, por eles favorecerem o acesso a alimentos não recomendados na dieta.

Assim, percebeu-se que diversas são as implicações causadas pelas restrições alimentares na vida diária de mulheres com DMG, e que estas incluem desde dificuldades de adaptação à nova dieta até privações importantes na vida diária, principalmente àquelas relacionadas aos momentos de lazer.

Aliada a essas constatações, a escassez de publicações sobre a temática dificulta o direcionamento de ações voltadas para as gestantes diabéticas, sendo uma limitação confirmada na presente pesquisa. Nesse âmbito, a fim de se elaborar estratégias e propostas de cuidados que auxiliem essas mulheres no enfrentamento dos obstáculos encontrados na gravidez de alto risco, principalmente aqueles relacionados às dificuldades com a dieta alimentar, recomenda-se a realização de estudos direcionados para esta importante parcela da população.

\section{REFERÊNCIAS}

1.American Diabetes Association. Diagnosis and classification of diabetes mellitus. Diabetes Care. 2014; 37 (supl 1):81-90.

2.Montenegro CAB. Rezende J. Obstetrícia fundamental. 13르 ed. Rio de Janeiro: Guanabara Koogan; 2014.

3.Varillas C, Blanco S, Couso B, Gastelu-Iturri J, Reboredo R. Diabetes gestacional: su complejidad y repercusión en la evolución del embarazo y salud del recién nacido. Progr Obst Ginecol. 2005; 48(6):289-96.

4.American Diabetes Association. Gestational diabetes mellitus. Diabetes Care. 2014; 37(9):103-5.

5.Ministério da Saúde (Br). Secretaria de Atenção à Saúde. Departamento de Ações Programáticas Estratégicas. Gestação de alto risco: manual técnico. $5^{\mathrm{a}}$ ed. Brasília (DF): Ministério da Saúde; 2010. 
6.Massucatti LA, Pereira RA, Maioli TU. Prevalência de diabetes gestacional em unidades de saúde básica. REAS. 2012;1(1):70-9.

7.Dode MASO, Santos IS. Fatores de risco para diabetes mellitus gestacional na coorte de nascimentos de Pelotas, Rio Grande do Sul, Brasil, 2004. Cad Saúde Pública. 2009; 25:1141-52. 8.Ryan EA. Hormones and insulin resistance during pregnancy. The Lancet. 2003; 362 (9398): 1777-8. 9.Oppermann MLR, Reichelt AJ. Diabetes melito e gestação. In: Freitas F, Martins-Costa SH, Ramos JGL, Magalhães, JA. Rotinas em Obstetrícia. 6a ed. Porto Alegre (RS): Artmed; 2011.

10.Ricci SS. Enfermagem materno-neonatal e saúde da mulher. Rio de Janeiro: Guanabara Koogan; 2008.

11. Tamez RN. Enfermagem na UTI neonatal: assistência ao recém-nascido de alto risco. $5^{\text {a }}$ ed. Rio de Janeiro: Guanabara Koogan; 2013.

12.Cavassini ACM, Lima SAM, Calderon IMP, Rudge MVC. Care cost for pregnant and

parturient women with diabetes and mild hyperglycemia. Rev Saude Publica. 2012; 46:334-43.

13.Luna NSA, Baeza MR, Castell EC, Santos FC, David HL, Castillo MMA. Intervención educativa: implementación de la agencia de autocuidado y adherencia terapéutica desde la

perspectiva del paciente diabético. Rev enferm UERJ. 2013; 21:289-94.

14.Schmalfuss JM, Prates LA, Azevedo M, Schneider $\mathrm{V}$. Diabetes melito gestacional e as implicações para o cuidado de enfermagem no pré-natal. Cogitare Enferm. 2014;19:815-22
15.Manoel MF, Marcon SS, Baldissera VDA. Estratégias educativas para pessoas com hipertensão arterial e diabetes mellitus. Rev enferm UERJ. 2013;21:403-8.

16.Bardin L. Análise de conteúdo. 2ª reimp. São Paulo: Edições 70; 2011.

17.Ministério da Saúde (Br). Conselho Nacional de Saúde. Comissão Nacional de Ética em Pesquisa. Resolução CNS no 466/2012. Brasília (DF): Ministério da Saúde; 2012.

18.Corrêa FHS, Gomes MB. Acompanhamento ambulatorial de gestantes com diabetes mellitus no Hospital Universitário Pedro Ernesto-UERJ. Arq Bras Endocrinol Metabol. 2004; 48:499-504.

19.Moretto VL, Lautert L. Características de gestantes portadoras de diabete melito. Rev Gaúcha Enferm. 2004; 25: 334-45.

20.Zugaib M. Obstetrícia. Barueri (SP): Manole; 2012. 21.Padilha PC, Sena AB, Nogueira JL, Araújo RPS, Alves PD, Accioly E, et al. Terapia nutricional no diabetes gestacional. Rev Nutr. 2010; 23(1):95-105.

22.Castillo CAG, Vásquez ML. El cuidado de sí de la embarazada diabética como uma via para asegurar um hijo sano. Texto contexto - enferm. 2006; 15(1):74-81. 23.Landim CAP, Milomens KMP, Diógenes MAR. Déficits de autocuidado em clientes com diabetes mellitus gestacional: uma contribuição para a enfermagem. Rev Gaúcha Enferm. 2008; 29:374-81.

24.Silva L, Santos RC, Parada CMGL. Compreendendo o significado da gestação para grávidas diabéticas. Rev Latino-Am Enfermagem. 2004; 12:899-904. 